\title{
NEW INEQUALITIES FOR OPERATOR CONCAVE FUNCTIONS INVOLVING POSITIVE LINEAR MAPS
}

\section{Shiva Sheybani, Mohsen Erfanian Omidvar and Hamid ReZa Moradi}

Abstract. The purpose of this paper is to present some general inequalities for operator concave functions which include some known inequalities as a particular case. Among other things, we prove that if $A \in \mathscr{B}(\mathscr{H})$ is a positive operator such that $m I \leqslant A \leqslant M I$ for some scalars $0<m<M$ and $\Phi$ is a normalized positive linear map on $\mathscr{B}(\mathscr{H})$, then

$$
\begin{aligned}
\left(\frac{M+m}{2 \sqrt{M m}}\right)^{r} & \geqslant\left(\frac{\frac{1}{\sqrt{M m}} \Phi(A)+\sqrt{M m} \Phi\left(A^{-1}\right)}{2}\right)^{r} \\
& \geqslant \frac{\frac{1}{(M m)^{\frac{r}{2}}} \Phi(A)^{r}+(M m)^{\frac{r}{2}} \Phi\left(A^{-1}\right)^{r}}{2} \\
& \geqslant \Phi(A)^{r} \sharp \Phi\left(A^{-1}\right)^{r},
\end{aligned}
$$

where $0 \leqslant r \leqslant 1$, which nicely extend the operator Kantorovich inequality. Mathematics subject classification (2010): Primary 47A63, Secondary 47À64, 15A60.

Keywords and phrases: Operator concave, operator inequalities, positive linear map, Kantorovich inequality, Bellman inequality.

\section{REFERENCES}

[1] T. ANDO, Concavity of certain maps on positive definite matrices and applications to Hadamard products, Linear Algebra Appl. 26 (1979), 203-241.

[2] T. ANdo AND F. HiAi, Operator log-convex functions and operator means, Math. Ann. 350, 3 (2011), 611-630.

[3] R. Bellman, On an inequality concerning an indefinite form, Amer. Math. Monthly. 63 (1956), 108-109.

[4] R. Bhatia, Positive definite matrices, Princeton (NJ): Princeton University Press; 2007.

[5] E. Y. LEE, A matrix reverse Cauchy-Schwarz inequality, Linear Algebra Appl. 430, 2 (2009), 805810.

[6] M. Lin, On an operator Kantorovich inequality for positive linear maps, J. Math. Anal. Appl. 402, 1 (2013), 127-132.

[7] A. W. Marshall And I. OlKin, Matrix versions of Cauchy and Kantorovich inequalities, Aequationes Math. 40 (1990), 89-93.

[8] J. MićIĆ, J. PEČARIĆ, Y. SEO AND M. TOMINAGA, Inequalities for positive linear maps on Hermitian matrices, Math. Inequal. Appl. 3, 4 (2000), 559-591.

[9] H. R. Moradi, M. E. OMIdvar, I. H. GÜMÜş AND R. NASERI, A note on some inequalities for positive linear maps, Linear Multilinear Algebra. 66, 7 (2018), 1449-1460.

[10] A. Morassaei, F. Mirzapour and M. S. Moslehian, Bellman inequality for Hilbert space operators, Linear Algebra Appl. 438, 10 (2013), 3776-3780.

[11] M. S. Moslehian, R. NAKAmoto AND Y. SEO, A Diaz-Metcalf type inequality for positive linear maps and its applications, Electron. J. Linear Algebra. 22 (2011), 179-190.

[12] R. NAKAmoto And M. NAKAMURA, Operator mean and Kantorovich inequality, Math. Japon. 44, 3 (1996), 495-498. 